\title{
Durable response to lenalidomide in a patient with myelodysplastic syndrome associated with isolated $5 q$ deletion and $J A K 2$ V617F mutation despite discontinuation of treatment
}

\author{
ELEFTHERIA HATZIMICHAEL ${ }^{1,2}$, KONSTANTINOS LAGOS $^{1}$, AMALIA VASSOU $^{1}$, \\ DORA GOUGOPOULOU $^{1}$, ALEXANDRA PAPOUDOU-BAI ${ }^{3}$ and EVANGELOS BRIASOULIS ${ }^{1}$ \\ ${ }^{1}$ Department of Hematology, University of Ioannina, 45110 Ioannina, Greece; ${ }^{2}$ Thomas Jefferson University, \\ Computational Medicine Center, Sidney Kimmel Medical College, Philadelphia, PA 19107, USA; \\ ${ }^{3}$ Department of Pathology, University Hospital of Ioannina, 45500 Ioannina, Greece
}

Received September 7, 2015; Accepted January 20, 2016

DOI: $10.3892 / \mathrm{mco} .2016 .866$

\begin{abstract}
Loss of a section of the long arm of chromosome 5, as a sole cytogenetic abnormality, characterizes a rare type of myelodysplastic syndrome [del(5q) MDS] and the co-existence of the JAK2 V617F mutation occurs in a small subset of these cases. Patients with isolated $\operatorname{del}(5 \mathrm{q})$ MDS have a relatively favorable prognosis, with transformation to acute myeloid leukemia occurring in $<10 \%$, and their disease responds well to lenalidomide. However the optimal therapeutic approach for patients with $\operatorname{del}(5 \mathrm{q}) \mathrm{MDS}$ in coexistence with the $J A K 2 \mathrm{~V} 617 \mathrm{~F}$ mutation, which is common to myeloproliferative neoplasms, remains to be elucidated. The present study reports a 77-year-old, transfusion-dependent female patient diagnosed with del(5q) MDS and a concomitant JAK2 V617F mutation. The patient was started on $10 \mathrm{mg}$ lenalidomide daily for 21 days in a 28 day-cycle and within the first month of treatment, the patient became transfusion-independent. The only toxicity observed was grade 3 neutropenia, which was managed with transient treatment discontinuation and dose reduction on restart $(5 \mathrm{mg})$. The patient achieved a complete cytogenetic and molecular response (normal karyotype and undetected JAK2 V617F mutation) within 6 months of treatment. However, 12 months post treatment initiation and while on hematological, cytogenetic and molecular response, the patient was unwilling to continue on treatment and lenalidomide was discontinued. The patient remains in hematological response, which lasts for $>5$ years despite treatment discontinuation. The present case highlights the coexistence of the $J A K 2 \mathrm{~V} 617 \mathrm{~F}$ mutation in $\operatorname{del}(5 \mathrm{q}) \mathrm{MDS}$ and suggests that lenalidomide treatment is beneficial and effective for these patients, leading to complete hematological,
\end{abstract}

Correspondence to: Professor Evangelos Briasoulis, Department of Haematology, University of Ioannina, P.O. Box 1186, 45110 Ioannina, Greece

E-mail: ebriasou@me.com

Key words: myelodysplastic syndrome, $\operatorname{del}(5 \mathrm{q})$, JAK2 V617F, lenalidomide cytogenetic and molecular response. Hematological response may be sustained for long periods of time, even following the discontinuation of the treatment.

\section{Introduction}

Deletion of the long arm of chromosome 5 is the most common cytogenetic abnormality in patients with myelodysplastic syndrome (MDS), occurring in $\sim 15 \%$ of cases (1). However, loss of the chromosome $5 \mathrm{q} 31.3$ to $5 \mathrm{q} 33.3$ region, as a sole chromosomal abnormality, is observed in $5 \%$ of MDS patients and characterizes $\operatorname{del}(5 \mathrm{q}) \mathrm{MDS}$, which is a clonal stem cell disorder (2). This MDS subtype has a favorable prognosis with transformation to acute myeloid leukemia occurring in $<10 \%$ of the cases and it responds well to lenalidomide treatment (3).

The $J A K 2$ V617F mutation is present in the majority of patients with myeloproliferative neoplasms (MPN): $>90 \%$ of patients with polycythemia vera, and $50 \%$ of patients with primary myelofibrosis and essential thrombocythemia (ET) (4). The presence of this mutation has also been described in other hematological malignancies, however, to a much lesser extent; $\sim 3-5 \%$ of patients with MDS and $<5 \%$ of patients with acute myeloid leukemia (AML) (5). The coexistence of $\operatorname{del}(5 q)$ as sole cytogenetic abnormality and the JAK2 V617F mutation is even rarer occurring in a small subset of del(5q) MDS cases, and the optimal therapeutic approach in these patients remains to be elucidated (6).

The present study reports the case of a patient with $\operatorname{del}(5 q)$ MDS and a concomitant JAK2 V617F mutation, who achieved complete cytogenetic and molecular remission with lenalidomide and enjoyed durable hematological response that lasts for $>5$ years post treatment discontinuation.

\section{Case report}

A 77-year-old female patient was referred to the Department of Haematology, University Hospital of Ioannina (Ioannina, Greece) in November 2008 for severe macrocytic anemia (hemoglobin, $6.5 \mathrm{~g} / \mathrm{dl}$ ), leucocytosis [white blood cell count (WBC), $\left.21.4 \times 10^{9} / 1\right]$ with predominance of the neutrophils and 
severe eosinophilia, and thrombocytosis (platelets, $774 \times 10^{9} / 1$ ). In the peripheral blood smear basophilic stippling and megaloblastoid changes of erythrocytes were observed. The biochemical tests were unremarkable, with the exception of increased levels of lactic dehydrogenase. On clinical examination, mild hepatosplenomegaly was observed, which was confirmed by ultrasound scan.

The patient underwent diagnostic assessments to exclude infections, connective tissue disorders and cancer. These assessments were negative. Computer tomography of the abdomen revealed mild hepatosplenomegaly. At this point bone marrow aspiration and biopsy were performed. Karyotyping and molecular analysis were also performed for the presence of $b c r-a b l$ chimeric transcript and JAK2 V617F mutation, and also FIP1L1/PDGFRA and TEL/PDGFRB rearrangements, considering MPN as the most likely diagnosis.

The bone marrow aspirate revealed increased cellularity, myeloid lineage hyperplasia with a left shift and increased numbers of hypolobated megakaryocytes, compatible with a MPN. Molecular analyses were positive for the presence of the $J A K 2$ V617F mutation and negative for the presence of $b c r-a b l$ transcript. Pending the results from the trephine biopsy and karyotyping, and on the basis of MPN being the most possible diagnosis, either ET or the cellular phase of primary myelofibrosis, a clinical decision was made to initiate treatment. The patient was started on interferon- $\alpha$, low-dose aspirin and recombinant erythropoietin as a result of a constant requirement for blood transfusions and her age, which defined her as a high-risk patient. A month later, WBC count and platelets were normalized, however, the patient remained transfusion-dependent, requiring at least two packed red blood cell units per month.

One month later, the results of karyotyping and the trephine biopsy were acquired. The latter was reported as having increased cellularity $(95 \%)$ with concomitant hypoplasia of the erythroid lineage; the myeloid lineage was hyperplastic with a left shift and the megakaryocytes were increased in number, decreased in size and their nuclei were hypolobulated, while micromegakaryocytes were also observed (Fig. 1). An abnormal karyotype was observed in all twenty metaphases that showed a deletion of the long arm of chromosome 5, involving the chromosome region 5q13-5q33 [46, XX, del (5) (q13 q33)], as the sole cytogenetic abnormality.

Therefore, the diagnosis, according to the WHO classification of hematologic neoplasms (7), was MDS with an isolated deletion of 5q chromosome and a concomitant JAK2 V617F mutation, categorizing her to the low risk group, according to the International Prognostic Scoring System (anemia, blasts $<5 \%$, favorable karyotype) (8). On the basis of definite diagnosis, previous therapy was discontinued and the patient was initiated on $10 \mathrm{mg}$ lenalidomide daily for 21 days in a 28-day-cycle since the patient was transfusion-dependent.

Within the first month of treatment the patient became transfusion-independent. The only toxicity observed was grade 3 neutropenia, and was managed by treatment withhold until resolution and one dose level reduction on restart $(5 \mathrm{mg})$. On lenalidomide $(5 \mathrm{mg})$ in cycles of $21 / 28$ days, the patient achieved both cytogenetic and molecular complete response (normal karyotype and undetected JAK2 V617F mutation) within 6 months of treatment (Fig. 2) and remained transfu- sion-independent, maintaining hemoglobin levels of $\sim 15 \mathrm{~g} / \mathrm{dl}$ for the next 6 months. However, 12 months post-treatment initiation and while on hematological, cytogenetic and molecular response, the patient was unwilling to continue with treatment and lenalidomide was discontinued. The patient remained in complete response for a further 23 months when a new bone marrow aspirate and cytogenetic analysis was performed and the karyotype of the diagnosis [46, XX, del (5) (q13 q33)] re-occurred, but in one of the twenty-four metaphases examined. However, the patient preserved the hematological and molecular response, and the transfusion-independency, therefore, treatment was not re-initiated. Currently, 70 months following diagnosis and 55 months without treatment, the patient is in excellent condition and transfusion-independent, maintaining a hematocrit level of $42 \%$.

\section{Discussion}

Approximately 5\% of MDS patients present with isolated del $(5 q)$, characterized by severe anemia, preserved or even elevated platelet count and high response rates to lenalidomide (3). The concomitant presence of the $5 \mathrm{q}$ deletion and a $J A K 2$ V617F mutation in MDS was first shown in 6/97 patients with MDS with the $5 \mathrm{q}$ deletion (6). Patients harboring the $J A K 2$ V617F mutation compared to those without the mutation, presented with hypercellular bone marrow and exhibited significantly higher levels of WBC count and a trend towards higher levels of platelet count (6). Since the clinical outcome of this subset of patients remains unclear, the 2008 WHO classification classifies them as MDS with isolated del(5q), rather than in the MDS/MPN category. Therefore, more data collection from patients with such characteristics are required in order to draw clear conclusions.

The present patient was diagnosed with isolated del(5q) MDS and a concurrent JAK2 V617F mutation. Lenalidomide was administered and the patient achieved transfusion-independence within 4 weeks of treatment initiation, and complete cytogenetic and molecular response within 6 months. The patient remains transfusion-independent for the last 70 months, despite lenalidomide discontinuation after 12 months of therapy. The del(5q) clone reappeared 23 months later in 1/24 metaphases, however, without clinical effects.

Beneficial treatment with lenalidomide in isolated del $(5 q)$ and a concomitant $J A K 2$ V617F mutation has been previously reported in three MDS patients (9-11) and one patient with AML arising from JAK2 V617F and del(5q) MDS. It remains unclear whether the lenalidomide effect has a suppressive role against del(5q) cells and another independent activity against the JAK2 V617F mutated clone (10), since it has not been delineated whether the two genetic abnormalities involve the same or two distinct hematopoietic clones. Sokol et al (12) investigated the clonal origin of the JAK2 V617F mutation in a patient with del(5q) MDS presenting with thrombocytosis and normal hemoglobin, and suggested that the latter is the case.

Currently, the recommendation regarding lenalidomide treatment in MDS del $(5 q)$ patients is to treat until disease progression (13). Although it was recently shown that lenalidomide treatment in MDS del(5q) patients does not appear to increase the risk to AML transformation (14), other long-term effects of immunomodulation have not been elucidated and 


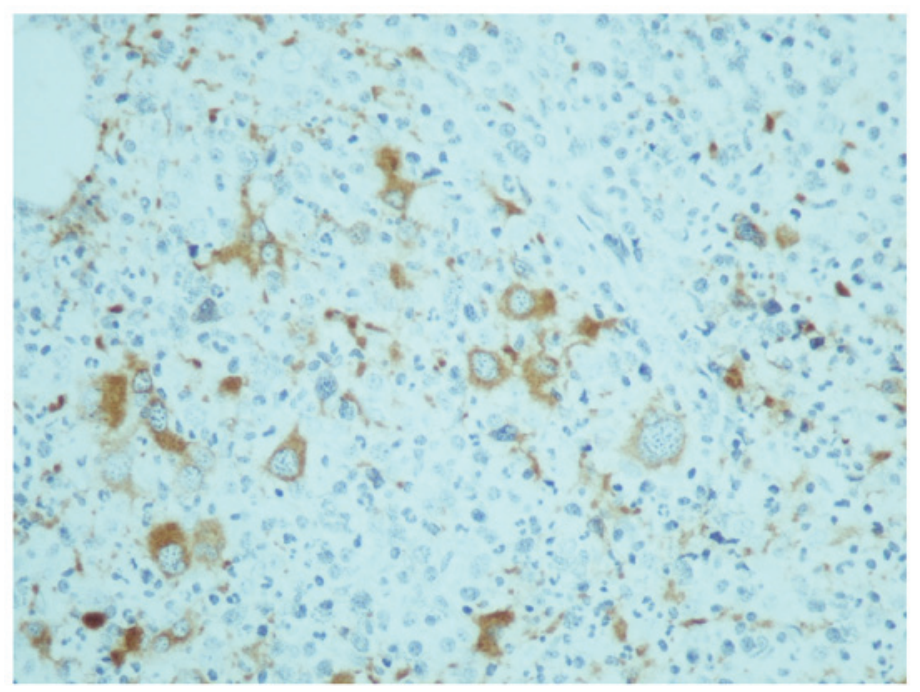

Figure 1. Immunohistochemical staining with cluster of differentiation 61 antibody revealed several megakaryocytes with hypolobated nuclei in bone marrow biopsy (magnification x400).

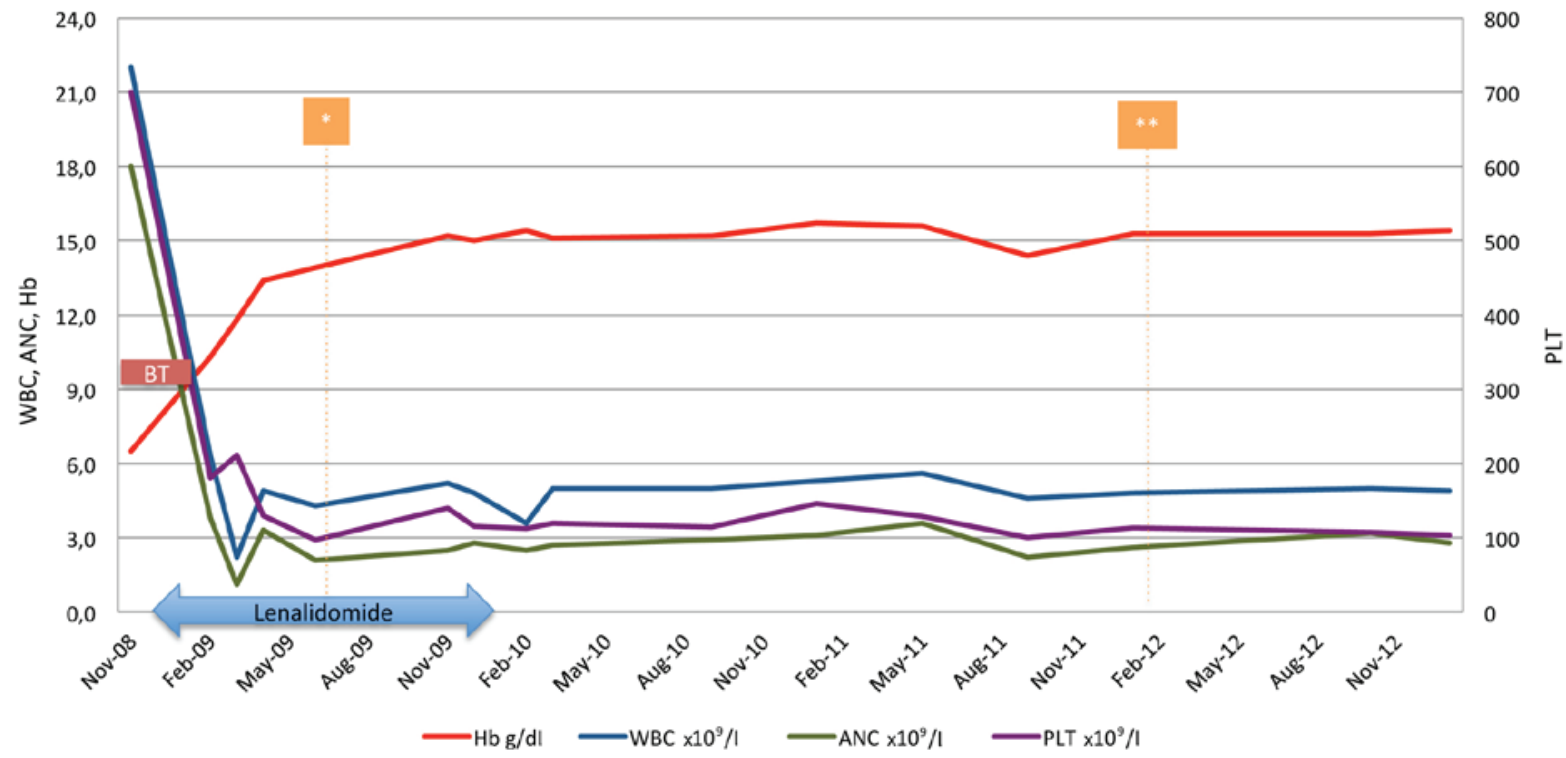

Figure 2. Peripheral blood counts prior to, during and following treatment with lenalidomide. The $y$-axis on the right represents WBC $\left(\mathrm{x} 10^{9} / 1\right), \mathrm{ANC}\left(\mathrm{x} 10^{9} / 1\right)$ and hemoglobin level $(\mathrm{g} / \mathrm{dl})$, and on the left PLT $\left(\mathrm{x} 10^{9} / \mathrm{l}\right)$. The blue arrow indicates the duration of lenalidomide therapy and the red box indicates the duration of BT. The orange boxes depict time points when cytogenetic analysis was performed ["documentation of normal karyotype and JAK2 V617F elimination; ** re-occurrence of del (5) (q13 q33) detected in 1/24 metaphases]. Hb, hemoglobin; WBC, white blood cell count; ANC, absolute neutrophils count; PLT, platelet count; BT, blood transfusions.

one could argue that intermittent use of lenalidomide may be prudent is certain cases. The present case indicated that durable hematological response may be sustained despite discontinuation of lenalidomide, whereas others have shown that re-challenge may also be safe and feasible, albeit with no profound cytogenetic response (15). However, in order to draw safe conclusions regarding the treatment duration and time of discontinuation, a randomized clinical trial is required to compare continuous treatment until progression, vs. treatment discontinuation after having achieved sustained complete cytogenetic response.

In conclusion, the present case highlights the coexistence of a JAK2 V617F mutation in $\operatorname{del}(5 \mathrm{q})$ MDS and suggests that lenalidomide treatment is beneficial and effective for this subset of patients, leading to complete cytogenetic and molecular response, while durable hematological response may be sustained even following the discontinuation of the treatment.

\section{References}

1. Bejar R, Levine R and Ebert BL: Unraveling the molecular pathophysiology of myelodysplastic syndromes. J Clin Oncol 29: 504-515, 2011 .

2. Cazzola M: Myelodysplastic syndrome with isolated $5 \mathrm{q}$ deletion (5q-syndrome). A clonal stem cell disorder characterized by defective ribosome biogenesis. Haematologica 93: 967-972, 2008.

3. List A, Dewald G, Bennett J, Giagounidis A, Raza A, Feldman E, Powell B, Greenberg P, Thomas D, Stone R, et al: Lenalidomide in the myelodysplastic syndrome with chromosome $5 \mathrm{q}$ deletion. N Engl J Med 355: 1456-1465, 2006. 
4. Baxter EJ, Scott LM, Campbell PJ, East C, Fourouclas N, Swanton S, Vassiliou GS, Bench AJ, Boyd EM, Curtin N, et al: Acquired mutation of the tyrosine kinase JAK2 in human myeloproliferative disorders. Lancet 365: 1054-1061, 2005.

5. Scott LM, Campbell PJ, Baxter EJ, Todd T, Stephens P, Edkins S, Wooster R, Stratton MR, Futreal PA and Green AR: The V617F JAK2 mutation is uncommon in cancers and in myeloid malignancies other than the classic myeloproliferative disorders Blood 106: 2920-2921, 2005.

6. Ingram W, Lea NC, Cervera J, Germing U, Fenaux P, Cassinat B, Kiladjian JJ, Varkonyi J, Antunovic P, Westwood NB, et al: The JAK2 V617F mutation identifies a subgroup of MDS patients with isolated deletion $5 \mathrm{q}$ and a proliferative bone marrow. Leukemia 20: 1319-1321, 2006.

7. Swerdlow SH, Campo E, Harris NL, Jaffe ES, Pileri SA, Stein H, Thiele J and Vardiman JW: WHO Classification of Tumours of Haematopoietic and Lymphoid Tissues. IARC Press, Lyon, France, 2008.

8. Greenberg P, Cox C, LeBeau MM, Fenaux P, Morel P, Sanz G, Sanz M, Vallespi T, Hamblin T, Oscier D, et al: International scoring system for evaluating prognosis in myelodysplastic syndromes. Blood 89: 2079-2088, 1997.

9. Azaceta G, Calasanz MJ, Dourdil V, Bonafonte E, Izquierdo I and Palomera L: Response to lenalidomide in a patient with myelodysplastic syndrome with isolated del $(5 q)$ and JAK2 V617F mutation. Leuk Lymphoma 51: 1941-1943, 2010.

10. Nomdedeu M, Maffioli M, Calvo X, Martínez-Trillos A, Baumann T, Díaz-Beyá M, Aguilar JL, Rozman M, Costa D, Esteve $\mathrm{J}$, et al: Efficacy of lenalidomide in a patient with myelodysplastic syndrome with isolated del(5q) and JAK2V617F mutation. Leuk Res 35: 1276-1278, 2011.

11. Musto P, Simeon V, Guariglia R, Bianchino G, Grieco V, Nozza F, La Rocca F, Marziano G, Lalinga AV, Fabiani E, et al Myelodysplastic disorders carrying both isolated del(5q) and JAK2 (V617F) mutation: Concise review, with focus on lenalidomide therapy. Onco Targets Ther 7: 1043-1050, 2014.
12. Sokol L, Caceres G, Rocha K, Stockero KJ, Dewald DW and List AF: JAK2 (V617F) mutation in myelodysplastic syndrome (MDS) with del(5q) arises in genetically discordant clones. Leuk Res 34: 821-823, 2010

13. Giagounidis A, Fenaux P, Mufti GJ, Muus P, Platzbecker U, Sanz G, Cripe L, Von Lilienfeld-Toal M and Wells RA: Practical recommendations on the use of lenalidomide in the management of myelodysplastic syndromes. Ann Hematol 87: 345-352, 2008

14. Adès L, Le Bras F, Sebert M, Kelaidi C, Lamy T, Dreyfus F, Eclache V, Delaunay J, Bouscary D, Visanica S, et al: Treatment with lenalidomide does not appear to increase the risk of progression in lower risk myelodysplastic syndromes with $5 \mathrm{q}$ deletion. A comparative analysis by the groupe francophone des myelodysplasies. Haematologica 97: 213-218, 2012.

15. Pisani F, Orlandi G and Merola R: Long-term response in a patient with del $(5 q)$ myelodysplastic syndrome who discontinued lenalidomide and obtained a good response and tolerance to rechallenge. Case Rep Oncol 7: 277-284, 2014. 